\title{
The Effect of Gene Mutations on Metastasis and Overall Survival in Metastatic and Nonmetastatic Colon Cancers
}

\author{
Yesim Ozdemir ${ }^{1 *}$, Murat $\mathrm{Cag}^{2}$, Emel Colak $^{3}$, Nuriye Coskun ${ }^{3}$, Neslihan Başgöz ${ }^{3}$, \\ Hakan Sarici ${ }^{4}$, Dilek Kaan ${ }^{5}$, Muhammet Dogan ${ }^{3}, K^{2}$ al Deniz ${ }^{6}$, Mevlide İnanc ${ }^{7}$, \\ Yusuf Ozkul ${ }^{3}$
}

\begin{abstract}
Objective: It is known that many genes are associated with colon cancer. We aimed to investigate the effect of gene mutations on metastasis and overall survival in metastatic and non metastatic colon cancers. Methods: A total of 50 patients with metastatic $(\mathrm{n}=25)$ and non metastatic $(\mathrm{n}=25)$ diagnosed with colon cancer between 2010 and 2018 were included in the study. APC, MUTYH, RAD50, MEN1, ATM, PALB2, NSH2, BRCA1, BRCA2, MLH1, BRIP1, TP53, $P T E N, B A R D 1, M S H 6, P M S 2, N B N$, and FAM175A gene mutations were evaluated using the next generation sequencing method. The effect of gene mutations on metastasis and overall survival were evaluated. Results: The mean age of patients with colon cancer without distant metastasis was $48.64 \pm 14.72$ years and for patients with distance metases was $56.68 \pm 11.65$. The mean survival time of colon cancer patients with distant organ metastasis after the metastasis date was $104.36 \pm 58.59$ weeks. The presence of $A P C, M U T Y H$, and TP53 genetic mutations was observed with a higher rate in metastatic colon cancer $(\mathrm{p}<0.05)$. Conclusion: We showed that APC, MUTYH, and TP53 mutations are associated with distant organ metastasis.
\end{abstract}

Keywords: Colon cancer- metastasis- gene mutuation

Asian Pac J Cancer Prev, 22 (12), 3839-3846

\section{Introduction}

Colorectal cancer (CRC), with more than 1.8 million new cases diagnosed in 2018, is one of the most common cancers worldwide (Schrembs et al, 2018). CRC is the second most common cancer in women $(9.2 \%)$ and third cancer in men $(10 \%)$ and CRC cases $(55 \%)$ are more common in Western countries (Marmol et al., 2017). The incidence of CRC has increased from 1990 to 2012, with the number of new cases exceeding the previous year's new cases by 200,000 cases (Brody et al., 2015). Improvement in survival in CRC has been observed due to efforts spent on screening and early detection and advances in systemic and local treatment methods (Schrembs et al., 2018). However, CRC is the fourth most common cause of cancer-related deaths, causing an average of 700,000 deaths per year (Marmol et al., 2017).

Identifying individual risk factors for the development of CRC and even risk factors causing local or distant metastasis and recurrence is very important as it can provide prognostic information for clinicians $(\mathrm{Xu}$ et al., 2020) CRC was one of the first tumors in which the various genes and pathways involved in the development and progression of the disease were investigated in detail (Fear on et al., 2011; Vogelstein et al., 1988; Vogelstein et al., 2013) From a genomic perspective, it is assumed that colorectal cancer is not a single disease, but a heterogeneous group of malignancies occurring in the colon. Therefore, genomic analysis of CRC will provide important prognostic information. Critical pathways include $A P C$, TP53, KRAS, NRAS, BRAF, BRCA1/2, $B M P R 1 A$, Her2, PIK3CA, transforming growth factor (TFG)- $\beta$, and mismatch repair (MMR) genes $M L H 1$, MSH2, MSH6, and PMS2 (Pearlman et al., 2016).

The major cause of death from CRC is development of metastases. When CRCs are diagnosed, approximately $22 \%$ are metastatic, and approximately $70 \%$ of patients will eventually develop metastatic relapse (Le Voyer et al., 2003; Markl et al., 2013). Considering that most of the deaths in CRC are caused by metastatic cancer, it is

\footnotetext{
${ }^{1}$ Uskudar University, Medical Faculty, Medical Genetics Department, Umraniye, 34768 Istanbul, Turkey. ${ }^{2}$ Université de Strasbourg, Service de chirurgie vasculaire et de transplantation, Nouvel Hôpital Civil, Université de Strasbourg 1 Place de l'Hôpital 67000 Strasbourg, France. ${ }^{3}$ Erciyes University, Medical Faculty, Medical Genetics Department, 38280, Kayseri, Turkey. ${ }^{4}$ Yozgat City Hospital,66100, Yozgat, Turkey. ${ }^{5}$ Betul- Ziya Eren Genome and Stem Cell Center, 38280, Kayseri, Turkey. ${ }^{6}$ Erciyes University, Medical Faculty, Pathology Department, 38280, Kayseri, Turkey. ${ }^{7}$ Erciyes University, Medical Faculty, Oncology Department,38280, Kayseri, Turkey.*For Correspondence: sevdayesimozdemir@gmail.com
} 
of vital importance to diagnose patients with CRC early and to reveal the factors that affect this process (Van der Geest et al., 2015).

To date, risk factors for CRC (increased BMI, red meat intake, cigarette smoking, low physical activity, low vegetable consumption, low fruit consumption etc.) (Johnson et al., 2013) are well defined. However, few studies investigate the effects of these risk factors on distant metastasis and overall survival. In this study, we aimed to detect germline mutations in 18 genes by Next-Generation sequencing in metastatic and nonmetastatic colon cancers and to investigate the effect of these mutations on metastasis and overall survival.

\section{Materials and Methods}

The institutional human study review committees of the Erciyes University 2019/794 approved this retrospective case-control study. This study was conducted in accordance with the tenets of the Declaration of Helsinki. In this study, a total of 50 patients with metastatic $(\mathrm{n}=25)$ and nonmetastatic $(\mathrm{n}=25)$ colon cancer who were followed up in Erciyes University Medical Oncology Department between 2010 and 2018 were included in the study. Overall survival was calculated as the time from diagnosis to death or to the last follow-up date.

\section{Next-Generation sequencing}

DNA was extracted from the sections taken from 5 micron thick paraffin blocks according to the manufacturer's instructions using QIAamp DNA FFPE Tissue kit. Sequence analysis was performed on the DNA. All variants reported here are detected with $>99 \%$ confidence, depending on the frequency of the mutation present and the amplicon extent. Mutations were analyzed in Sophia DDM program using a new generation sequencing kit containing 18 genes $(A P C$, MUTYH, RAD50, MEN1, ATM, PALB2, NSH2, BRCA1, BRCA2, MLH1, BRIP1, TP53, PTEN, BARD1, MSH6, PMS2, NBN, FAM175A).

\section{Statistical Analysis}

The data were evaluated using the IBM SPSS Statistics Standard Concurrent User V 25 (IBM Corp., Armonk, New York, USA) statistical program. For descriptive statistics, unit number (n), percent (\%), mean \pm standard deviation $\left(\mathrm{x}^{-} \pm \mathrm{ss}\right)$, median (M), smallest value (min), largest value $(\max )$ are given as values. The normal distribution of data for post-metastasis survival of patients with age and colon cancer patients with distant organ metastasis was evaluated using Shapiro-Wilk normality test and Q-Q charts. For the age variable, the difference between groups with and without distant organ metastasis was evaluated by independent samples t-test. The relationship between the presence of genetic mutation status and smoking status, gender, grade, cancer stage, lymph node metastasis, family history, tumor location, disease classification, and patient survival categorical variables were examined using the Exact method of the chi-square test in $2 \times 2$ and $\mathrm{r} x \mathrm{c}$ tables. Subgroup analyses were performed using Bonferroni corrected two-rate tests. Post-metastasis survival (weeks) of patients with colon cancer with distant organ metastasis was determined. A regression model was created, and Cox regression analysis was performed to determine the prognostic and predictive values of factors affecting the life span of patients with colon cancer with distant organ metastasis. A p $<0.05$ value was considered statistically significant.

\section{Results}

The mean age of patients with colon cancer without distant organ metastasis $(n=25)$ included in the study $48.64 \pm 14.72$ (Min: 15, Max: 75) years, and the mean age of patients with colon cancer with distant organ metastasis $(n=25)$ was $56.68 \pm 11.65$ (Min: 30, Max: 73) years. The mean age of patients without distant organ metastasis was lower than those with distant organ metastasis $(p=0.037)$. The average life span of colon cancer patients with distant organ metastasis after the date of metastasis was found to be 104.36 \pm 58.59 (Min: 22, Max: 267) weeks.

The frequency distributions, percentages, and the relationship between variables according to the genetic mutation status of all colon cancer patients are given in Table 1. A significant relationship was found between the groups with the presence of APC, MUTYH, and TP53 genetic mutations $(\mathrm{p}<0.05)$. The presence of APC and MUHTY genetic mutations is 3.083 times higher in patients with colon cancer with distant organ metastasis compared to patients with colon cancer without distant organ metastasis (OR $=3.083,95 \% \mathrm{CI}$ : 1.937-4.909). Similarly, the presence of TP53 genetic mutations is 6,364 times higher in patients with colon cancer with distant organ metastasis than in patients with colon cancer without distant organ metastasis $(\mathrm{OR}=2.125,95 \% \mathrm{CI}$ : 1.488-3.035). There were no significant differences in the presence of other genetic mutations and between the groups $(\mathrm{p}>0.05)$.

The results of the effects of genetic mutations of all colon cancer patients on overall survival are given in Table 2. There was not a significant relationship between the presence of genetic mutation and overall survival ( $p>$ $0.05)$.

The effects of sociodemographic and clinical characteristics on overall survival of patients with colon cancer without distant organ metastasis are given in Table 3. No significant relationships were found between smoking, gender, stage, family history, tumor location and pathogenicity with disease status categories ( $\mathrm{p}>$ 0.05). According to Table 4, no significant correlation was found between the presences of genetic mutations on overall survival in patients with colon cancer without distant organ metastasis $(\mathrm{p}>0.05)$.

The sociodemographic and clinical characteristics of patients with colon cancer with distant organ metastasis and the effects of genetic mutations on overall survival are given in Table 5 and Table 6. No significant relationships were found between smoking, gender, stage, family history, tumor location, pathogenicity, presence of genetic mutation and overall survival ( $\mathrm{p}>0.05)$.

A significant relationship was found between the presence of $A P C, M U T H Y$, and $N S H 2$ genetic mutations 
DOI:10.31557/APJCP.2021.22.12.3839

The Effect of Gene Mutations on Metastasis

Table 1. Frequency Distributions, Percentages and Relationships with Metastasis in All Patients with Colon Cancer by Genetic Mutation Status

\begin{tabular}{|c|c|c|c|c|c|c|c|}
\hline \multirow{2}{*}{\multicolumn{4}{|c|}{$\begin{array}{l}\text { Relationships with Metastasis in All Patients with Colon } \\
\text { Cancer by Genetic Mutation Status }\end{array}$}} & \multirow{3}{*}{ Variables } & \multirow{3}{*}{$\begin{array}{l}\text { Without distant } \\
\text { organ metastasis } \\
(\mathrm{n} / \%)\end{array}$} & \multirow{3}{*}{$\begin{array}{l}\text { With distant } \\
\text { organ metastasis } \\
(\mathrm{n} / \%)\end{array}$} & \multirow{3}{*}{$\begin{array}{c}\mathrm{p} \\
\text { value }\end{array}$} \\
\hline & & & & & & & \\
\hline \multirow[t]{2}{*}{ Variables } & \multirow{2}{*}{$\begin{array}{l}\text { Without distant } \\
\text { organ metastasis } \\
(\mathrm{n} / \%)\end{array}$} & \multirow{2}{*}{$\begin{array}{l}\text { With distant } \\
\text { organ metastasis } \\
(\mathrm{n} / \%)\end{array}$} & \multirow{2}{*}{$\begin{array}{c}\mathrm{p} \\
\text { value }\end{array}$} & & & & \\
\hline & & & & \multicolumn{4}{|l|}{$N B N$} \\
\hline \multicolumn{4}{|l|}{$A P C$} & No & - & $1(4)$ & $1.000 \%$ \\
\hline No & $6(33.3)^{\mathrm{a}}$ & $-{ }^{b}$ & \multirow[t]{2}{*}{$0.003 \ddagger$} & \multicolumn{4}{|l|}{ FAM175A } \\
\hline Yes & $12(66.7)^{\mathrm{a}}$ & $25(100)^{b}$ & & No & - & $1(4)$ & $1.000 \%$ \\
\hline MUTYH & & & & Yes & $18(100)$ & $24(96)$ & \\
\hline $\begin{array}{l}\text { No } \\
\text { Yes } \\
\text { RAD50 }\end{array}$ & $\begin{array}{l}6(33.3)^{\mathrm{a}} \\
12(66.7)^{\mathrm{a}}\end{array}$ & $\begin{array}{c}-b \\
25(100)^{b}\end{array}$ & $0.003 \ddagger$ & $\begin{array}{l}*, \text { Column } \mathrm{p} \\
a \text { and } b \text { shou } \\
\text { same letter a }\end{array}$ & $\begin{array}{l}\text { tages are indicated; } \\
\text { lifference between gy } \\
\text { illar. }\end{array}$ & $\begin{array}{l}\text { isher Exact Test; S } \\
\text { ups. The distributio }\end{array}$ & $\begin{array}{l}\text { iperscripts } \\
\text { ls with the }\end{array}$ \\
\hline No & $1(5.6)$ & - & $0.419 \ddagger$ & & & & \\
\hline $\begin{array}{c}\text { Yes } \\
\text { MEN1 }\end{array}$ & $17(94.4)$ & $25(100)$ & & $\begin{array}{l}\text { Table } 2 . \\
\text { Overall } \mathrm{S} \\
\text { Included } \mathrm{i}\end{array}$ & $\begin{array}{l}\text { mination of th } \\
\text { val and Genetic } \\
\text { Study }\end{array}$ & $\begin{array}{l}\text { Relationship } \\
\text { Iutations of All }\end{array}$ & $\begin{array}{l}\text { between } \\
\text { Patients }\end{array}$ \\
\hline No & $1(5.6)$ & $1(4)$ & $1.000 \ddagger$ & Variables & Overall Su & ival & \\
\hline Yes & $17(94.4)$ & $24(96)$ & & & Alive $(n / \%)$ & Dead (n/\%) & $\mathrm{p}$ value \\
\hline$A T M$ & & & & $A P C$ & & & \\
\hline No & $3(16.7)$ & $4(16)$ & $1.000 \ddagger$ & No & $5(22.7)$ & $1(4.8)$ & $0.185 \ddagger$ \\
\hline Yes & $15(83.3)$ & $21(84)$ & & Yes & $17(77.3)$ & $20(95.2)$ & \\
\hline$P A L B 2$ & & & & MUTYH & & & \\
\hline No & $1(5.6)$ & - & $0.419 \ddagger$ & No & $5(22.7)$ & $1(4.8)$ & $0.185 \ddagger$ \\
\hline Yes & $17(94.4)$ & $25(100)$ & & Yes & $17(77.3)$ & $20(95.2)$ & \\
\hline $\mathrm{NSH} 2$ & & & & RAD50 & & & \\
\hline No & $3(16.7)$ & $1(4)$ & $0.293 \ddagger$ & No & $1(4.5)$ & - & $1.000 \%$ \\
\hline Yes & $15(83.3)$ & $24(96)$ & & Yes & $21(95.5)$ & $21(100)$ & \\
\hline$B R C A 1$ & & & & MEN1 & & & \\
\hline No & $1(5.6)$ & - & $0.419 \ddagger$ & No & $1(4.5)$ & $1(4.8)$ & $1.000 \ddagger$ \\
\hline Yes & $17(94.4)$ & $25(100)$ & & Yes & $21(95.5)$ & $20(95.2)$ & \\
\hline$B R C A 2$ & & & & $A T M$ & & & \\
\hline No & $2(11.1)$ & - & $0.169 \ddagger$ & No & $4(18.2)$ & $3(14.3)$ & $1.000 \ddagger$ \\
\hline Yes & $16(88.9)$ & $25(100)$ & & Yes & $18(81.8)$ & $18(85.7)$ & \\
\hline MLHI & & & & $P A L B 2$ & & & \\
\hline No & - & $1(4)$ & $1.000 \ddagger$ & No & $1(4.5)$ & - & $1.000 \ddagger$ \\
\hline Yes & $18(100)$ & $24(96)$ & & Yes & $21(95.5)$ & $21(100)$ & \\
\hline BRIPI & & & & $\mathrm{NSH} 2$ & & & \\
\hline No & - & $1(4)$ & $1.000 \ddagger$ & No & $3(3.6)$ & $1(4,8)$ & $0.607 \ddagger$ \\
\hline Yes & $18(100)$ & $24(96)$ & & Yes & $19(86.4)$ & $20(95,2)$ & \\
\hline TP53 & & & & $B R C A 1$ & & & \\
\hline No & $-{ }^{a}$ & $9(36)^{b}$ & $0.006 \$$ & No & $1(4.5)$ & - & $1.000 \ddagger$ \\
\hline Yes & $18(100)^{\mathrm{a}}$ & $16(64)^{b}$ & & Yes & $21(95.5)$ & $21(100)$ & \\
\hline PTEN & & & & $B R C A 2$ & & & \\
\hline No & - & $2(8)$ & $0.502 \ddagger$ & No & $2(9.1)$ & - & $0.488 \ddagger$ \\
\hline Yes & $18(100)$ & $23(92)$ & & Yes & $20(90.9)$ & $21(100)$ & \\
\hline$B A R D 1$ & & & & $M L H 1$ & & & \\
\hline No & - & $1(4)$ & $1.000 \ddagger$ & No & $1(4.5)$ & - & $1.000 \ddagger$ \\
\hline Yes & $18(100)$ & $24(96)$ & & Yes & $21(95.5)$ & $21(100)$ & \\
\hline MSH6 & & & & BRIP1 & & & \\
\hline No & - & $3(12)$ & $0.252 \ddagger$ & No & $1(4.5)$ & - & $1.000 \ddagger$ \\
\hline Yes & $18(100)$ & $22(88)$ & & Yes & $21(95.5)$ & $21(100)$ & \\
\hline$P M S 2$ & & & & TP53 & & & \\
\hline No & - & $1(4)$ & $1.000 \ddagger$ & No & $2(9.1)$ & $7(33.3)$ & $0.069 \ddagger$ \\
\hline Yes & $18(100)$ & $24(96)$ & & Yes & $20(90.9)$ & 14 (66.7) & \\
\hline
\end{tabular}

Table 1. Continued

Table 2. Examination of the Relationship between Overall Survival and Genetic Mutations of All Patients ncluded in the Study 
Table 2. Continued

\begin{tabular}{|c|c|c|c|}
\hline \multirow[t]{2}{*}{ Variables } & \multicolumn{2}{|c|}{ Overall Survival } & \multirow[b]{2}{*}{$\mathrm{p}$ value } \\
\hline & Alive (n/\%) & $\operatorname{Dead}(\mathrm{n} / \%)$ & \\
\hline \multicolumn{4}{|l|}{ PTEN } \\
\hline No & - & $2(9.5)$ & $0.233 \ddagger$ \\
\hline Yes & $22(100)$ & $19(90.5)$ & \\
\hline \multicolumn{4}{|l|}{$B A R D 1$} \\
\hline No & - & $1(4.8)$ & $0.488 \ddagger$ \\
\hline Yes & $22(100)$ & $20(95.2)$ & \\
\hline \multicolumn{4}{|l|}{ MSH6 } \\
\hline No & $2(9.1)$ & $1(4.8)$ & $1.000 \%$ \\
\hline Yes & $20(90.9)$ & $20(95.2)$ & \\
\hline \multicolumn{4}{|l|}{$P M S 2$} \\
\hline No & - & $1(4.8)$ & $0.488 \div$ \\
\hline Yes & $22(100)$ & $20(95.2)$ & \\
\hline \multicolumn{4}{|l|}{$N B N$} \\
\hline No & $1(4.5)$ & - & $1.000 \%$ \\
\hline Yes & $21(95.5)$ & $21(100)$ & \\
\hline \multicolumn{4}{|l|}{ FAM175A } \\
\hline No & $1(4.5)$ & - & $1.000 \mathrm{ts}$ \\
\hline Yes & $21(95.5)$ & $21(100)$ & \\
\hline
\end{tabular}

*, Column percentages are indicated; \$, Fisher Exact Test

and cancer stage (Table 7) $(p=0.014 ; p=0.014 ; p=0.016)$. There were no significant relationships between the presence of other genetic mutations categories and cancer stage categories $(p>0.05)$. The relationship between each

Table 3. Investigation of the Relationship between Sociodemographic and Clinic Pathological Characteristics of Patients with Colon Cancer without Distant Organ Metastasis on Overall Survival

\begin{tabular}{lccc}
\hline Variables & \multicolumn{3}{c}{ Overall Survival } \\
& Alive (n/\%) & Dead (n/\%) & p value \\
\hline Smoking status & $10(47,6)$ & $2(50)$ & $1,000 \ddagger$ \\
$\quad$ No & $11(52,4)$ & $2(50)$ & \\
Yes & & & \\
Gender & $10(47,6)$ & $2(50)$ & $1,000 \ddagger$ \\
$\quad$ Female & $11(52,4)$ & $2(50)$ & \\
Male & & & \\
Staging & $6(54,5)$ & - & - \\
II & $4(36,4)$ & - & \\
III & $1(9,1)$ & - & \\
IV & & & \\
Family story & $16(80)$ & $2(100)$ & $1,000 \ddagger$ \\
No & $4(20)$ & - & \\
$\quad$ Yes & & & \\
Tumor Location & $3(21,4)$ & $1(100)$ & $0,267 \ddagger$ \\
Right column & $11(78,6)$ & - & \\
Left column & & & \\
Classification & $9(81,8)$ & $1(100)$ & $1,000 \ddagger$ \\
Significance unknown & $2(18,2)$ & - & \\
Pathogenic / Possibly & & & \\
pathogenic & & & \\
\hline
\end{tabular}

*, Column percentages are indicated; $\$$, Fisher Exact Test
Table 4. The Relationship of Patients with Colon Cancer without Distant Organ Metastases with Overall Survival According to Their Genetic Mutation Status

\begin{tabular}{|c|c|c|c|}
\hline \multirow[t]{2}{*}{ Variables } & \multicolumn{2}{|c|}{ Overall Survival } & \multirow[b]{2}{*}{$\mathrm{p}$ value } \\
\hline & Alive (n/\%) & $\operatorname{Dead}(\mathrm{n} / \%)$ & \\
\hline \multicolumn{4}{|l|}{$A P C$} \\
\hline No & $5(31.3)$ & $1(50)$ & $1.000 \ddagger$ \\
\hline Yes & $11(68.7)$ & $1(50)$ & \\
\hline \multicolumn{4}{|l|}{ MUTYH } \\
\hline No & $5(31.3)$ & $1(50)$ & $1.000 \dagger$ \\
\hline Yes & $11(68.7)$ & $1(50)$ & \\
\hline \multicolumn{4}{|l|}{ RAD50 } \\
\hline No & $1(6.3)$ & - & $1.000 \ddagger$ \\
\hline Yes & $15(93.7)$ & $2(100)$ & \\
\hline \multicolumn{4}{|l|}{ MEN1 } \\
\hline No & $1(6.3)$ & - & $1.000 \ddagger$ \\
\hline Yes & 15 (93.7) & $2(100)$ & \\
\hline \multicolumn{4}{|l|}{$A T M$} \\
\hline No & $2(12.5)$ & $1(50)$ & $0.314 \ddagger$ \\
\hline Yes & $14(87.5)$ & $1(50)$ & \\
\hline \multicolumn{4}{|l|}{$P A L B 2$} \\
\hline No & $1(6.3)$ & - & $1.000 \dagger$ \\
\hline Yes & $15(93.7)$ & $2(100)$ & \\
\hline \multicolumn{4}{|l|}{$\mathrm{NSH} 2$} \\
\hline No & $3(18.7)$ & - & $1.000 \ddagger$ \\
\hline Yes & $13(81.3)$ & $2(100)$ & \\
\hline \multicolumn{4}{|l|}{$B R C A 1$} \\
\hline No & $1(6.3)$ & - & $1.000 \ddagger$ \\
\hline Yes & 15 (93.7) & $2(100)$ & \\
\hline \multicolumn{4}{|l|}{$B R C A 2$} \\
\hline No & $2(12.5)$ & - & $1.000 \ddagger$ \\
\hline Yes & $14(87.5)$ & $2(100)$ & \\
\hline \multicolumn{4}{|l|}{$M L H 1$} \\
\hline No & - & - & - \\
\hline Yes & $16(100)$ & $2(100)$ & \\
\hline \multicolumn{4}{|l|}{ BRIPI } \\
\hline No & - & - & - \\
\hline Yes & $16(100)$ & $2(100)$ & \\
\hline \multicolumn{4}{|l|}{ TP53 } \\
\hline No & - & - & - \\
\hline Yes & $16(100)$ & $2(100)$ & \\
\hline \multicolumn{4}{|l|}{ PTEN } \\
\hline No & - & - & - \\
\hline Yes & $16(100)$ & $2(100)$ & \\
\hline \multicolumn{4}{|l|}{ BARDI } \\
\hline No & - & - & - \\
\hline Yes & $16(100)$ & $2(100)$ & \\
\hline \multicolumn{4}{|l|}{ MSH6 } \\
\hline No & - & - & - \\
\hline Yes & $16(100)$ & $2(100)$ & \\
\hline \multicolumn{4}{|l|}{ PMS2 } \\
\hline No & - & - & - \\
\hline Yes & $16(100)$ & $2(100)$ & \\
\hline
\end{tabular}


Table 4. Continued

\begin{tabular}{lccc}
\hline Variables & \multicolumn{2}{c}{ Overall Survival } & \\
& Alive $(\mathrm{n} / \%)$ & Dead $(\mathrm{n} / \%)$ & $\mathrm{p}$ values \\
\hline$N B N$ & & & \\
No & - & - & - \\
Yes & $16(100)$ & $2(100)$ & \\
FAM175A & & & - \\
No & - & - & \\
Yes & $16(100)$ & $2(100)$ & \\
\hline
\end{tabular}

*, Column percentages are indicated; $\ddagger$, Fisher Exact Test

genetic mutation status and gender, smoking status was examined, and no significant relationships were found $(\mathrm{p}>0.05)$.

A significant relationship was found between the stage and overall survival status of patients with colon cancer without distant organ metastasis and with distant organ metastasis. The duration of life (weeks) after metastasis of colon cancer patients with distant organ metastasis was taken as the dependent variable. Cox regression analysis was performed to determine the prognostic and predictive values. It was found that age has a significant effect in determining the survival time for the colon cancer patient group with distant organ metastasis (Age $=1.110 ; 95 \%$ CI: $1.011-1.129 ; \mathrm{p}=0.028)$.

Table 5. The Relationship between Sociodemographic and Clinic Pathological Characteristics of Patients with Colon Cancer with Distant Organ Metastasis on Overall Survival

\begin{tabular}{|c|c|c|c|}
\hline \multirow[t]{2}{*}{ Variables } & \multicolumn{2}{|c|}{ Overall Survival } & \multirow[b]{2}{*}{$\mathrm{p}$ value } \\
\hline & Alive (n/\%) & Dead (n/\%) & \\
\hline \multicolumn{4}{|l|}{ Smoking status } \\
\hline No & $4(66.7)$ & $9(47.4)$ & $0.645 \ddagger$ \\
\hline Yes & $2(33.3)$ & $10(52.6)$ & \\
\hline \multicolumn{4}{|l|}{ Gender } \\
\hline Female & $3(50)$ & $7(36.8)$ & $0.653 \ddagger$ \\
\hline Male & $3(50)$ & $12(63.2)$ & \\
\hline \multicolumn{4}{|l|}{ Staging } \\
\hline II & $1(16.7)$ & - & $0.290 \ddagger$ \\
\hline III & - & $2(10.5)$ & \\
\hline IV & $5(83.3)$ & $17(89.5)$ & \\
\hline \multicolumn{4}{|l|}{ Family History } \\
\hline No & $4(66.7)$ & $15(100)$ & $0.071 \ddagger$ \\
\hline Yes & $2(33.3)$ & - & \\
\hline \multicolumn{4}{|l|}{ Tumor Location } \\
\hline Right column & $4(66.7)$ & $8(53.3)$ & $0.659 \ddagger$ \\
\hline Left column & $2(33.3)$ & 7 (46.7) & \\
\hline \multicolumn{4}{|l|}{ Classification } \\
\hline $\begin{array}{l}\text { Significance } \\
\text { unknown }\end{array}$ & - & $1(11.1)$ & $1.000 \%$ \\
\hline $\begin{array}{c}\text { Pathogenic/ } \\
\text { Possibly pathogenic }\end{array}$ & $4(100)$ & $8(88.9)$ & \\
\hline
\end{tabular}

Table 6. Relationship between Genetic Mutation Status and Overall Survival of Patients with Colon Cancer with Distant Organ Metastasis

\begin{tabular}{|c|c|c|c|}
\hline \multirow[t]{2}{*}{ Variables } & \multicolumn{2}{|c|}{ Overall Survival } & \multirow[b]{2}{*}{$p$ values } \\
\hline & Alive (n/\%) & Dead (n/\%) & \\
\hline \multicolumn{4}{|l|}{$A P C$} \\
\hline No & - & - & - \\
\hline Yes & $6(100)$ & $19(100)$ & \\
\hline \multicolumn{4}{|l|}{ MUTYH } \\
\hline No & - & - & - \\
\hline Yes & $6(100)$ & $19(100)$ & \\
\hline \multicolumn{4}{|l|}{ RAD50 } \\
\hline No & - & - & - \\
\hline Yes & $6(100)$ & $19(100)$ & \\
\hline \multicolumn{4}{|l|}{ MEN1 } \\
\hline No & - & $1(5.3)$ & $1.000 \%$ \\
\hline Yes & $6(100)$ & $18(94.7)$ & \\
\hline \multicolumn{4}{|l|}{$A T M$} \\
\hline No & $2(33.3)$ & $2(10.5)$ & $0.234 \%$ \\
\hline Yes & $4(66.7)$ & $17(89.5)$ & \\
\hline \multicolumn{4}{|l|}{$P A L B 2$} \\
\hline No & - & - & - \\
\hline Yes & $6(100)$ & $19(100)$ & \\
\hline \multicolumn{4}{|l|}{$\mathrm{NSH} 2$} \\
\hline No & - & $1(5.3)$ & $1.000 \%$ \\
\hline Yes & $6(100)$ & $18(94.7)$ & \\
\hline \multicolumn{4}{|l|}{ BRCA1 } \\
\hline No & - & - & - \\
\hline Yes & $6(100)$ & $19(100)$ & \\
\hline \multicolumn{4}{|l|}{$B R C A 2$} \\
\hline No & - & - & - \\
\hline Yes & $6(100)$ & $19(100)$ & \\
\hline \multicolumn{4}{|l|}{$M L H 1$} \\
\hline No & $1(16.7)$ & - & $0.240 \%$ \\
\hline Yes & $5(83.3)$ & $19(100)$ & \\
\hline \multicolumn{4}{|l|}{ BRIPI } \\
\hline No & $1(16.7)$ & - & $0.240 \%$ \\
\hline Yes & $5(83.3)$ & $19(100)$ & \\
\hline \multicolumn{4}{|l|}{ TP53 } \\
\hline No & $2(33.3)$ & $7(36.8)$ & $1.000 \%$ \\
\hline Yes & $4(66.7)$ & $12(63.2)$ & \\
\hline \multicolumn{4}{|l|}{ PTEN } \\
\hline No & - & $2(10.5)$ & $1.000 \%$ \\
\hline Yes & $6(100)$ & $17(89.5)$ & \\
\hline \multicolumn{4}{|l|}{ BARDI } \\
\hline No & - & $1(5.3)$ & $1.000 \%$ \\
\hline Yes & $6(100)$ & $18(94.7)$ & \\
\hline \multicolumn{4}{|l|}{ MSH6 } \\
\hline No & $2(33.3)$ & $1(5.3)$ & $0.133 \$$ \\
\hline Yes & $4(66.7)$ & $18(94.7)$ & \\
\hline \multicolumn{4}{|l|}{ PMS2 } \\
\hline No & - & $1(5.3)$ & $1.000 \%$ \\
\hline Yes & $6(100)$ & $18(94.7)$ & \\
\hline
\end{tabular}


Table 6. Continued

\begin{tabular}{lccc}
\hline Variables & \multicolumn{3}{c}{ Overall Survival } \\
& Alive (n/\%) & Dead (n/\%) & $\mathrm{p}$ values \\
\hline$N B N$ & & & \\
No & $1(16.7)$ & - & $0.240 \ddagger$ \\
Yes & $5(83.3)$ & $19(100)$ & \\
FAM175A & & & \\
No & $1(16.7)$ & - & $0.240 \ddagger$ \\
Yes & $5(83.3)$ & $19(100)$ & \\
*, Column percentages are indicated; + Fisher Exact Test &
\end{tabular}

\section{Discussion}

CRC, about $56 \%$ of whom die of cancer, is the third most common cancer worldwide (Riihimaki et al.,2012). Both the frequency of CRC and its risks should be well defined in order to reduce the mortality rate from CRC. There are many habits or traits that increase the likelihood of developing colorectal cancer or polyp and are considered risk factors. Risk factors for CRC include age (Levin et al., 2008), inflammatory bowel disease (patients with ulcerative colitis have an $3.7 \%$ increased risk (Eaden et al., 2001) while people suffering from Crohn's disease have a $2.5 \%$ increased risk of developing CRC (Canavan et al., 2006) positive family history of CRC in relatives under the age of fifty (Johns et al., 2001), a sedentary lifestyle associated with obesity (Martinez-Useros et al., 2016), unhealthy eating habits(Willet et al., 2005).

Mutations may occur in tumor suppressor genes, oncogenes, and genes involved in DNA repair mechanisms in the initiation of CRC (Fear on et al., 1990). CRC can be classified as sporadic, hereditary, or familial. Sporadic cancers account for $70 \%$ of all CRC and are heterogeneous, as mutations in genes different from the molecular pathogenesis of sporadic cancer are targeted. $\mathrm{CRC}$ begins as a benign adenomatous intestinal polyp and turns into adenocarcinoma that can metastasize to distant organs. Each step in such gradual progression of CRC known as "multi-step tumorigenesis" is related to specific genetic changes in oncogenes or tumor suppressors (Fear on et al., 2011; Ferron et al., 1990; Markowitz et al., 2009). The development of polyps begins with a mutation in a tumor suppressor gene known as adenomatous polyposis coli (APC). Mutations in KRAS, TP53, and DCC follow the $A P C$ mutation. Hereditary polyposis cancer primarily includes familial adenomatous polyposis (FAP), while hereditary non-polyposis colorectal cancer (HNPCC) is associated with mutations in DNA repair mechanisms. Lynch syndrome is the most common cause of HNPCC. Inherited mutations in genes encoding DNA repair proteins such as MLH1, PMS1, MSH2, PMS2 and MLH6 are responsible for this syndrome (Lynch et al., 2003; Umar et al., 2004).

Although the genes involved in the development of CRC are well defined, there has not been much study on the genes that play a role in the development of distant organ metastasis and affect survival. Fang et al. (Fang et al., 2014) identified six genes ( $A D C Y 2$, $A D C Y 9, A P C, G N B 5, K R A S$, and $L R P O$ ) associated with CRC metastasis. $A P C, K R A S$, and $\beta$-catenin mutations
Table 7. Examination of the Frequency Distributions, Percentages and the Relationship with Cancer Stage of Patients with Distant Organ Metastasis and Colon Cancer without Distant Organ Metastasis According to Genetic Mutation Status

\begin{tabular}{ccccc}
\hline Variables & $\begin{array}{c}\text { Stage II } \\
(\mathrm{n} / \%)\end{array}$ & $\begin{array}{c}\text { Stage } \\
\text { Stage III } \\
(\mathrm{n} / \%)\end{array}$ & $\begin{array}{c}\text { Stage IV } \\
(\mathrm{n} / \%)\end{array}$ & p value \\
\hline APC & & & & \\
No & $1(16.7)^{a, b}$ & $2(40) \mathrm{v}$ & $-a$ & $0.014 \dagger$ \\
Yes & $5(83.3)^{a, b}$ & $3(60)^{b}$ & $23(100)^{a}$ & \\
MUTHY & & & & \\
No & $1(16.7)^{a, b}$ & $2(40)^{b}$ & $-\mathrm{a}$ & $0.014 \dagger$ \\
Yes & $5(83.3)^{a, b}$ & $3(60)^{b}$ & $23(100)^{a}$ & \\
NSH2 & & & & \\
No & $-a, b$ & $2(40)^{b}$ & $-{ }^{a}$ & $0.016 \dagger$ \\
Yes & $6(100)^{a, b}$ & $3(60)^{b}$ & $23(100)^{a}$ & \\
\hline
\end{tabular}

*, Column percentages are indicated; $\uparrow$, Pearson Exact Chi-Square Test; Superscripts a and b show the difference between groups. The distributions with the same letter are similar.

are important in the development of adenoma, and PIK3CA and TP53 mutations are especially important in the progression to invasive CRC (Lee et al., 2017). $K R A S$ mutations are associated with metastasis, poor prognosis, and lower survival (Chen et al., 2014; Li et al., 2015) Recent studies have reported a reduced overall survival in $B R A F$-mutated CRC (41.1 vs 18.2 months) (Caymanian et al., 2018). In another study, it was observed that the loss of PTEN in primary tumors in patients with metastatic CRC was significantly associated with an increased risk of death and poor survival (Atria et al., 2013) Decrease in BARD1 expression has been found to cause worse staging and therefore poor prognosis (Spurn et al., 2011). TP53 mutations were reported in previous studies to be associated with poor prognosis in various types of cancer, including CRC (Oliver et al., 2010). TP53 mutation rate increased to $80 \%$ in patients with metastatic CRC (Brannon et al., 2014). A TP53 mutation gradually increases from early stage tumors to metastasis (Yeager et al., 2018). Therefore, TP53 mutations play a role, especially in the late stage of tumorigenesis (Nakayama et al., 2019). Chang et al., (2016) showed that the frequency of NRAS mutation increased in patients with lung metastasis and that NRAS and TP53 were positively associated with tumor stage and prognosis. In our study, the presence of APC and MUTYH genetic mutations is 3.083 times greater in patients with colon cancer with distant organ metastasis compared to patients with colon cancer without distant organ metastasis. Similarly, patients with colon cancer with distant organ metastases exhibited 6.364 times more TP53 genetic mutations than patients with colon cancer without distant organ metastases. In previous studies, the relationships between APC and TP53 mutations and CRC metastasis have been shown, but as far as we know, this is the first study reporting that MUTYH affects CRC metastasis. In addition, in our study, we determined a relationship between APC, MUTYH, and $\mathrm{NSH} 2$ and tumor stage. As the stage of the tumor increased, the presence of APC, MUTYH, and NSH2 
mutations also increased. Therefore, APC and MUTYH have a bad effect on overall survival as they both increase distant organ metastasis and are associated with high stage, and TP53 is increased in distant organ metastasis. When Cox regression analysis was performed in determining prognostic and predictive values, we found that age had a significant effect in determining the survival time for the colon cancer patient group with distant organ metastasis.

In conclusion, we show that APC, MUTYH, and TP53 mutations are associated with distant organ metastasis. We also found a relationship between APC, MUTYH, and $\mathrm{NSH} 2$ and tumor stage. We found that age had a significant effect in determining the survival time for the colon cancer patient group with distant organ metastasis. The results of our study should be supported by larger studies.

\section{Author Contribution Statement}

YO and YO the idea for research. MC, YO and MD were the major contributors in literature search writing the manuscript, and spelling and grammar check. HS, EC and NG managed collecting tissues. MI, Referred the patients NB and DK conducting the DNA analysis and NGS. All authors read and approved the final manuscript.

\section{Acknowledgements}

\section{Ethics approval}

The institutional human study review committees of the Erinyes University 2019/794 approved this retrospective case-control study.

\section{Consent to participate}

This study was conducted in accordance with the tenets of the Declaration of Helsinki.

\section{Consent for publication}

All of the authors declare that they have all participated in the design, execution, and analysis of the paper, and that they have approved the final version

Availability of data and material not applicable

\section{Conflicts of interest}

The authors have no conflicts of interest to declare that are relevant to the content of this article.

\section{References}

Atreya CE, Sangale Z, Xu N, et al (2013). Pten expression is consistent in colorectal cancer primaries and metastases and associates with patient survival. Cancer Med, 2, 496-506.

Brannon AR, Vakiani E, Sylvester BE, et al (2014). Comparative sequencing analysis reveals high genomic concordance between matched primary and metastatic colorectal cancer lesions. Genome Biol, 15, 454.

Brody H (2015). Colorectal cancer. Nature, 521, 7551.

Canavan C, Abrams KR, Mayberry J (2006). Meta-analysis: colorectal and small bowel cancer risk in patients with crohn's disease. Aliment Pharmacol Ther, 23, 1097-104.

Chang SC, Lin PC, Lin JK et al (2016). Mutation spectra of common cancer-associated genes in different phenotypes of colorectal carcinoma without distant metastasis. Ann Surg
Oncol, 23, 849-55.

Chen J, Guo F, Shi X, et al (2014). Braf v600e mutation and kras codon 13 mutations predict poor survival in chinese colorectal cancer patients. BMC Cancer, 14, 802.

Eaden JA, Abrams KR, Mayberry JF (2001). The risk of colorectal cancer in ulcerative colitis: A meta-analysis. Gut, 48, 526-35.

Fang LT, Lee S, Choi H, et al (2014). Comprehensive genomic analyses of a metastatic colon cancer to the lung by whole exome sequencing and gene expression analysis. Int J Oncol, 44, 211-21.

Fearon ER, Vogelstein B (1990). A genetic model for colorectal tumorigenesis. Cell, 61, 759-67.

Fearon ER (2011). Molecular genetics of colorectal cancer. Annu Rev Pathol, 6, 479-507.

Johns LE, Houlston RS (2001). A systematic review and meta-analysis of familial colorectal cancer risk. Am J Gastroenterol Suppl, 96, 2992-3003.

Johnson C, Wei M, Ensor C, et al (2013). Meta-analyses of colorectal cancer risk factors. Cancer Causes Control, 24, 1207-22.

Kayhanian H, Goode E, Sclafani F, et al (2018). Treatment and survival outcome of braf-mutated metastatic colorectal cancer: a retrospective matched case-control study. Clin Colorectal Cancer, 17, 69-76.

Lee SH, Jung SH, Kim TM, et al (2017). Whole-exome sequencing identified mutational profiles of high-grade colon adenomas. Oncotarget, 8, 6579-88.

Le Voyer TE, Sigurdson ER, Hanlon AL, et al (2003). Colon cancer survival is associated with increasing number of lymph nodes analyzed: a secondary survey of intergroup trial int-0089. J Clin Oncol, 21, 2912-9.

Levin B, Lieberman DA, McFarland B, et al (2008). Screening and surveillance for the early detection of colorectal cancer and adenomatous polyps, 2008: a joint guideline from the american cancer society, the us multi-society task force on colorectal cancer, and the american college of radiology. $C A$ Cancer J Clin, 58, 130-60.

Li W, Qiu T, Zhi W, et al (2015). Colorectal carcinomas with kras codon 12 mutation are associated with more advanced tumor stages. BMC Cancer, 15, 340.

Lynch HT, de la Chapelle A (2003). Hereditary colorectal cancer. N Engl J Med, 348, 919-32.

Markl B, Schaller T, Krammer I, et al (2013). Methylene blueassisted lymph node dissection technique is not associated with an increased detection of lymph node metastases in colorectal cancer. Mod Pathol, 26, 1246-54.

Markowitz SD, Bertagnolli MM (2009). Molecular origins of cancer: molecular basis of colorectal cancer. $N$ Engl J Med, 361, 2449-60.

Marmol I, Sanchez-de-Diego C, Pradilla Dieste A (2017). Colorectal carcinoma: a general overview and future perspectives in colorectal cancer. Int J Mol Sci, 18, 197.

Martinez-Useros J, Garcia-Foncillas J (2016.) Obesity and colorectal cancer: molecular features of adipose tissue. J Transl Med, 14, 21.

Nakayama M, Oshima M (2019). Mutant p53 in colon cancer. J Mol Cell Biol, 11, 267-76.

Olivier M, Hollstein M, Hainaut P (2010). TP53 mutations in human cancers: origins, consequences, and clinical use. Cold Spring Harb Perspect Biol, 2, a001008.

Pearlman R, Frankel WL, Swanson B, et al (2017). Prevalence and spectrum of germline cancer susceptibility gene mutations among patients with early-onset colorectal cancer. JAMA Oncol, 3, 464-71.

Riihimaki M, Thomsen H, Sundquist K, Hemminki K (2012). Colorectal cancer patients: what do they die of?. Frontline 
Gastroenterol, 3, 143-9.

Schrembs P, Martin B, Anthuber M, Schenkirsch G, Markl B (2018). The prognostic significance of lymph node size in node-positive colon cancer. PLoS One, 13, e0201072.

Sporn JC, Hothorn T, Jung B (2011). Bard1 expression predicts outcome in colon cancer. Clin Cancer Res, 17, 5451-62.

Umar A, Boland CR, Terdiman JP, et al (2004). Revised bethesda guidelines for hereditary nonpolyposis colorectal cancer (lynch syndrome) and microsatellite instability. $J$ Natl Cancer Inst Monogr, 96, 261-8.

van der Geest LG, Lam-Boer J, Koopman M, et al (2015). Nationwide trends in incidence, treatment and survival of colorectal cancer patients with synchronous metastases. Clin Exp Metastasis, 32, 457-65.

Vogelstein B, Fearon ER, Hamilton SR, et al (1988). Genetic alterations during colorectal-tumor development. $N$ Engl $J$ Med, 319, 525-32.

Vogelstein B, Papadopoulos N, Velculescu VE, et al (2013). Cancer genome landscapes. Science, 339, 1546-58.

Willett WC (2005). Diet and cancer: an evolving picture. JAMA, 293, 233-4.

$\mathrm{Xu}$ W, He Y, Wang Y (2020). Risk factors and risk prediction models for colorectal cancer metastasis and recurrence: an umbrella review of systematic reviews and meta-analyses of observational studies. BMC Med, 18, 172.

Yaeger R, Chatila WK, Lipsyc MD, et al (2018). Clinical sequencing defines the genomic landscape of metastatic colorectal cancer. Cancer Cell, 33, 125-36.

This work is licensed under a Creative Commons AttributionNon Commercial 4.0 International License. 